\title{
Determining the heterogeneity in time of the dynamics within a slowly relaxing region of a supercooled liquid: Role of sharp relaxation events
}

\author{
L. M. Alarcón, M. A. Frechero, R. A. Montani, and G. A. Appignanesi* \\ Sección Fisicoquímica, INQUISUR-UNS-CONICET and Departamento de Química, Universidad Nacional del Sur, \\ Avenida Alem 1253, 8000 Bahía Blanca, Argentina \\ (Received 30 December 2008; revised manuscript received 24 June 2009; published 26 August 2009)
}

\begin{abstract}
Supercooled liquids have been shown to be dynamically heterogeneous with different regions of the system presenting dynamics that vary from each other even by orders of magnitude. Computer simulations have confirmed such a picture by detecting that the mobile particles in model glass formers are not homogeneously distributed within the system but arranged in clusters. More recently, the dynamics of small systems has been characterized by demonstrating that their structural relaxation is not homogeneous in time, in the sense that it does not evolve gradually but it is signed by rapid bursts of mobility characterized by relative compact clusters of mobile particles. These events (which have been named $d$ clusters) are fast and sparse and trigger the transitions the system experiences between metabasins (MB) of its potential-energy surface. The MB residence times are much larger than the time scales of occurrence of the $d$ clusters, and it has been suggested that the events that occur within them scarcely contribute to the structural relaxation of the system. Thus, the picture of glassy relaxation that emerges would indicate that at any time a supercooled liquid may present different spatial regions, each one characterized by different structural relaxation times. In turn, each of such regions would not relax smoothly or gradually but by means of sporadic sharp relaxation events. Here, we assess for a model glass former the relative relevance of the MB exploration events and of the $d$ clusters both in small systems and within regions of large systems, to show that the structural relaxation at the region level is indeed extremely heterogeneous in time and utterly governed by the latter.
\end{abstract}

DOI: 10.1103/PhysRevE.80.026127

PACS number(s): 61.20.Ja, 61.20.Lc

\section{INTRODUCTION}

The understanding of the molecular basis of the dynamical slowing down that emerges as a liquid is supercooled under its melting point represents a main issue in condensed matter [1-6]. This glassy regime is characterized by dynamics that vary by orders of magnitude from one region of the system to another [1-6] while the relaxation has been believed to proceed by means of cooperatively relaxing regions whose time scales and sizes grow considerably as the temperature is decreased [1-7]. The validity of a heterogeneous scenario has been confirmed both experimentally and theoretically, since the existence of dynamical heterogeneities [8-15] has been detected. Pioneering computational studies for an archetypical glass former (the Kob-Andersen binary Lennard-Jones mixture) identified that such system (for system sizes of $N=1000$ and $N=8000$ particles) is most heterogeneous at a given time scale, named $t^{*}$, when the dynamics significantly departs from that expected by a Brownian behavior [9]. Within such time scale, the dynamics has been shown to be characterized by the presence of cooperative motions of a small number of particles (a few percent) arranged in clusters and which move collectively, often in a stringlike fashion, by a distance close to the particle diameter [9]. This finding represented a confirmation that the dynamics of the system within a structural relaxation time $\left(\tau_{\alpha}\right.$, which is determined by the time when the incoherent selfintermediate scattering function has decayed to some arbitrarily small value: most authors use $1 / e$, as we shall use

\footnotetext{
*appignan@criba.edu.ar
}

here, while others consider the decay to $10 \%$ ) is clearly heterogeneous in space. However, such studies focused on the global displacement from $t=0$ to $t=t^{*}$ and the clusters observed need not develop gradually or concurrently. In fact, the study of the time evolution of the large stringlike clusters present within a large system indeed shows that they decompose in different small stringlike movements which occur at quite different times, thus indicating that the different regions of the system relax asynchronically [16,17]. More recently, focusing on small systems $(N=150)$, the fact that the dynamics of such systems is also quite heterogeneous in time has been suggested by the finding of collective motions of a significant fraction of the particles which form relatively compact clusters [18]. This heterogeneity in time means that the relaxation of such small systems is not homogeneous or gradual since the dynamics presents sharp bursts of mobility (that is, the trajectory is most of the time quite "inactive" to then suffer sharp jumps). These very rapid and sporadic events, which were termed "democratic motions" or $d$ clusters, drive the system from one metabasin (MB) of its potential-energy surface (a group of similar closely related structures $[3,18-20])$ to another, the time scale of the exploration of the MBs being much larger than the MB transition events, while the structural relaxation (the so-called $\alpha$ relaxation) is performed by a small number of such transition events. These cooperatively relaxing units or $d$ clusters have been identified in different glassy systems like the aforementioned binary Lennard-Jones (LJ) [18] system and supercooled water [21] and represent natural candidates for the cooperatively relaxing regions proposed long ago by Adam and Gibbs [7]. A recent inhomogeneous mode-coupling theory of dynamical heterogeneity has related them to the 
(fractal) geometrical structures carrying the dynamical correlations at time scales commensurable with that of the $\alpha$ relaxation [22]. Additionally, a recent experimental and computational work in a glassy polymer provided experimental support to the MB-MB transitions and $d$ clusters [23]. In such study [23], the jumps in the fluorescence of an isolated probe have been shown to be correlated with MB transitions and $d$-cluster-like events. Since the probe is sensitive to the movement and dipole change of its environment, the experiment senses the relaxation dynamics of a single (relatively small) region of the system and not the system as a whole and thus is directly comparable with our computational results $[18,23]$.

Differently from the situation in a small system, the dynamics in large systems does not show heterogeneity in time. That is, the relaxation of the global system does not show large inactive periods of times to evolve by means of sharp events. Thus, the global outcome is gradual (perfectly gradual in the thermodynamic limit). However, this does not necessarily mean that the differently relaxing regions within such a system are relaxing gradually, but that the times when they present their rapid sporadic relaxation events ( $d$ clusters) are different from that of the other regions. In other words, the behavior detected in small systems would still hold valid within small regions of the large system, but is averaged out when we consider the system as a whole. Thus, it is of interest to follow trajectories within a large system by focusing at each time on single relaxing regions. The studies in small systems of size comparable with the typical sizes of such regions could be instructive in this sense (systems of around a hundreds of particles would be useful since the sizes of the typical relaxing regions within a large system $[18,24]$ are expected to be close to that value for certain temperature regime within the supercooled regime; the size of such regions is expected to grow as temperature diminishes). However, the methods used in Ref. [18], at variance from the ones used in other studies aimed at detecting metabasin transitions and relevant relaxation events, are directly applicable to small-size subsystems immersed within a large one. Thus, one of the aims of this work is to study the relaxation dynamics of large systems at the region level.

Another interesting piece of information in this context is the demonstration that the structural constraints posed by the initial configuration on the dynamics do not survive a $d$ cluster and fade at time scales shorter than the $\alpha$-relaxation time [25]. Thus, the distribution of the propensity for motion of the particles (the tendency to be mobile when evaluated within the isoconfigurational ensemble, that is, for an ensemble of trajectories started form the same initial configuration but changing the initial particle velocities [26,27]) changes after a MB transition event, a fact also consistent with a recent appealing description of glassy relaxation in terms of the localized low-frequency normal (soft) modes [28]. This is so since many different MBs are available from any given MB. This result supports a picture of the $\alpha$ relaxation as a random walk on MBs which had been previously proposed given the lack of correlation found between successive MB waiting times [29].

All the above expounded findings suggest a scenario in which the local dynamics in a supercooled liquid (the dy- namics within different regions of a large system) passes through large periods of inactivity (in terms of the structural $\alpha$ relaxation) to suffer dynamical hot spots characterized by the occurrence of the $d$ clusters, which would thus account for most of the local structural relaxation. Thus, the aim of the present work is to explore the heterogeneity in time of the $\alpha$ relaxation both in small systems and within regions of a large system and to further assess the relative relevance of the different dynamical events. In this respect, our present results will demonstrate that the structural relaxation of the system is in fact almost exclusively due to the $d$ clusters. For instance, we shall show that both the mean-squared displacement (MSD) and the incoherent self intermediate scattering function evolve mainly by means of such events.

\section{MODEL SYSTEM AND METHODS}

\section{A. Dynamics of the binary Lennard-Jones system}

We performed a series of molecular dynamics (MD) simulations within the NVE microcanonical ensemble (that is, constant number of particles, $\mathrm{N}$, constant volume, $\mathrm{V}$, and constant energy, E) for a widely used model of fragile glass former: the binary Lennard-Jones system consisting of a three-dimensional mixture of $80 \% A$ and $20 \% \mathrm{~B}$ particles, the size of the $A$ particles being larger than the $B$ ones $[9,18,30]$. The interaction between two atoms of type $A$ and $B$ is given by $V_{\alpha \beta}(r)=4 \epsilon_{\alpha \beta}\left\{\left(\sigma_{\alpha \beta} / r\right)^{12}-\left(\sigma_{\alpha \beta} / r\right)^{6}\right\}$, where $\alpha, \beta \in\{A, B\}$. The LJ parameters used are $\epsilon_{A A}=1.0, \sigma_{A A}$ $=1.0, \epsilon_{A B}=1.5, \sigma_{A B}=0.8, \epsilon_{B B}=0.5$, and $\sigma_{B B}=0.88$. These interactions have been truncated and shifted at $r_{\text {cutoff }}=2.5 \sigma_{\alpha \beta}$. The equations of motion were solved for the NVE ensemble at a particle density of 1.2 , using the velocity form of the Verlet algorithm with a time step of 0.02. All the presented results correspond to the situation in equilibrium. To obtain the inherent structure (IS) of a given configuration we employed the conjugate-gradient method. We shall show results from systems at temperature $T=0.5$, density of 1.2 and $N$ $=150$ particles [18]. This system size avoids the interference of results from many different subsystems (metabasins) while being free of finite-size effects, as shown in Ref. [18]. We shall also study systems with $N=8000$ particles. However, in order to look for MBs and $d$ clusters at such temperature, one has to focus on the behavior of subsystems (of around 150 particles each) and thus the large system should be decomposed in many small subsystems wherein to apply the concept of $\mathrm{MB}[19,20]$. If not, one expects that the results from different regions of the system would average out the behavior expected for a single small region. Thus, besides its simplicity as compared to other methods, the advantage of the distance matrix (DM) method we shall employ, as described below, is that it makes it possible to study portions of any size from a large system since it relies only on particle coordinates and not potential energy. This has also the advantage that for systems of size arbitrarily small there is no need to modify the original potential of the Kob-Andersen model, as demanded by other methods. Given this fact, part of our work will be referred to a small system of $N=150$ and for some cases we shall also show results for a system of size $N=8000$ by focusing on small regions within it. At low tem- 
peratures (close and above the critical temperature predicted by the mode-coupling theory of the glass transition, $T_{\mathrm{c}}$ $=0.435)$ this system presents dynamical heterogeneities $[9,18]$ : there are many particles that move more than what would be expected on the basis of a Brownian behavior and a small number of particles move cooperatively at a distance that is comparable to the interparticle distance. These "fast moving" (or "mobile") particles are not homogeneously distributed throughout the sample but are arranged in clusters usually made of stringlike groups of particles $[9,18]$. The dynamics is most heterogeneous at time $t^{*}$ defined by the maximum in the non-Gaussian parameter $\alpha_{2}(t), \alpha_{2}(t)$ $=\frac{3\left\langle r^{4}(t)\right\rangle}{5\left\langle r^{2}(t)\right\rangle^{2}}-1$, which measures the deviation of the self part of the van Hove function $\left[G_{\mathrm{s}}(r, t)\right.$, the probability at a given time $t$ of finding a particle at distance $r$ from its initial position] from a Brownian behavior [9]. This quantity is located at the end of the $\beta$ relaxation and beginning of the $\alpha$ relaxation (the crossover from the caging to the diffusive regime in the mean-squared displacement $\left\langle r^{2}(t)\right\rangle$ plot) and constitutes the characteristic time for dynamical heterogeneities (in this case $t^{*}=400$ for $T=0.50$ [18]). Additionally, $t^{*}$ depends strongly on temperature and grows quickly as we move toward $T_{\mathrm{c}}[31]$. However, not all the mobile particles within a $t^{*}$ time span contribute decisively to the $\alpha$ relaxation, as we have recently demonstrated [18]. Instead, the $\alpha$ relaxation is driven by a series of a few MB-MB transitions which are triggered by the occurrence of large compact clusters of medium-range-mobility particles called democratic particles [18]. Additionally, the typical residence time in a MB has been estimated to be on the order of $t^{*}$ [18].

\section{B. Distance matrix method, metabasins, and $d$ clusters}

We now describe briefly the distance matrix method to study MB dynamics (see Refs. [18,25] for details): we perform an MD simulation and record equally spaced configurations (for example 101 configurations, as in Refs. $[18,25]$ ) for a time larger than that of the $\alpha$-relaxation time, $\tau_{\alpha}$. The total run time for the simulations is $t=4000$, while $\tau_{\alpha} \approx 700$ when evaluated as the time when the self intermediate scattering function for the first peak of the structure factor has decayed to $1 / e$ (the definition we shall use here as most authors do, while some other authors use the time scale of the decay of such function to $10 \%$, which in this case is around 2900). Thus, consecutively recorded configurations are separated in time by $\theta=40\left(=10 \% t^{*}\right)$ and build the following distance matrix $[18,32], R^{2}\left(t^{\prime}, t^{\prime \prime}\right)=\frac{1}{N} \sum_{i=1}^{N}\left|\mathbf{r}_{i}\left(t^{\prime}\right)-\mathbf{r}_{i}\left(t^{\prime \prime}\right)\right|^{2}$, where $\mathbf{r}_{i}(t)$ is the position of particle $i$ at time $t$. Thus, $R^{2}\left(t^{\prime}, t^{\prime \prime}\right)$ gives the system-averaged-squared displacement (ASD) of a particle in the time interval that starts at $t^{\prime}$ and ends at $t^{\prime \prime}$. In other words, this distance matrix contains the averaged-squared distances between each recorded configuration and all the other ones. For this study (as all studies dealing with MBs [18-20,32]), we must investigate small systems, since for large systems the results originated from different subsystems would obscure the conclusions [18-20,32]. Thus, we study now a system of $N=150$ particles. However, we also found the same qualitative results for small subsystems immersed in a big one, thus ruling out
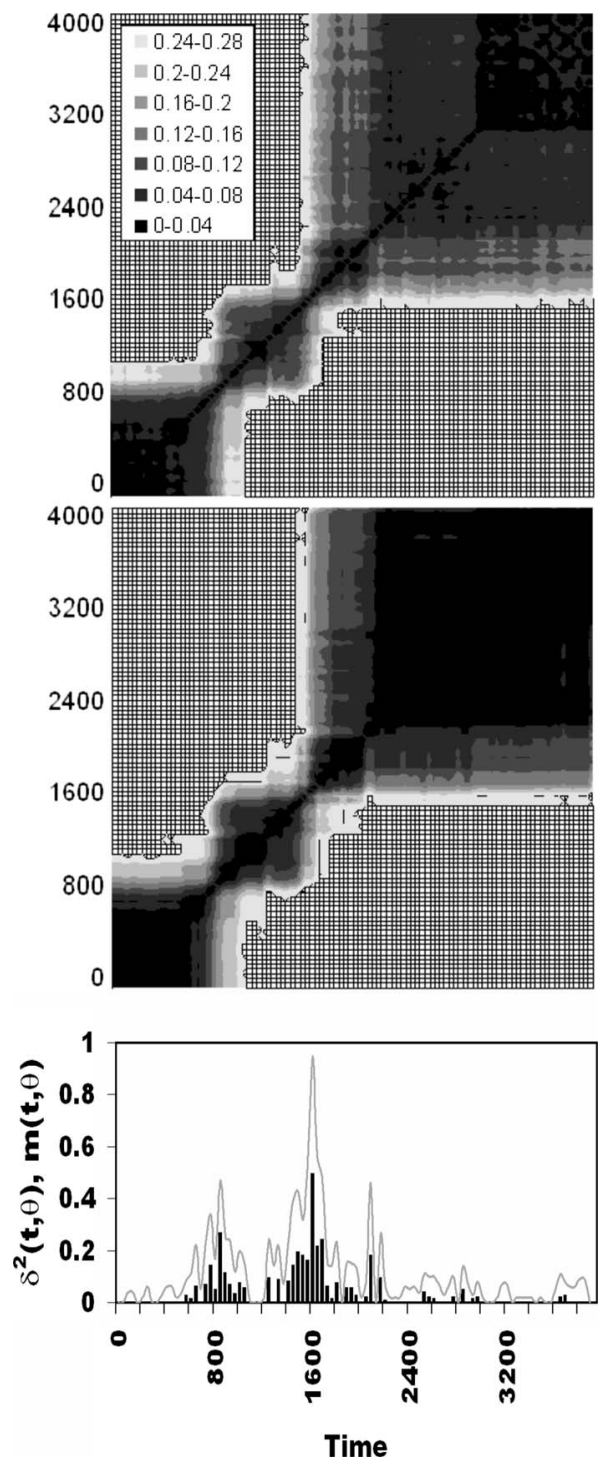

FIG. 1. Top: contour plot of the distance matrix $R^{2}\left(t^{\prime}, t^{\prime \prime}\right), \mathrm{DM}$, for $T=0.5$. The gray levels correspond to the values indicated. Middle: the distance matrix calculated at the inherent structure level. Note the clear similitude between the two DMs. Bottom: the averaged-squared displacement $\delta^{2}(t, \theta)$ for the same trajectory (gray line) and the function $m(t, \theta)$, which gives the fraction of democratic particles, i.e., particles that moved more than the threshold value $r_{t h}=0.25$ in the time interval $[t, t+\theta]$ (black bars). These curves were obtained for the inherent dynamics and $\theta=40$.

the possibility for finite-size effects (that is, we repeated the study for subsystems of 150 particles within a large system of 8000 particles, which means to focus on small portions of a large system).

Figure 1 (top) shows a typical behavior for trajectories with $T=0.5$. The gray level of the squares in $R^{2}\left(t^{\prime}, t^{\prime \prime}\right)$ depicts the distance between the corresponding configurations, the darker the shading indicating the lower the distance between them. Were the dynamics homogeneous in time, we would expect a distance matrix with a dark main diagonal and a continuous fading as we move away from such diagonal. However, from the island structure of this matrix a clear MB structure of the landscape is evident. That is, islands are 
made up of closely related configurations (low $R^{2}$ ) which are separated from the configurations of other islands by large distances. In other words, the dynamics of the system is inhomogeneous in time. We can estimate the typical residence time in the MBs for this $T$ (from island sizes) as qualitatively on the order of $t^{*}$. Given the small system size we expect this to be a good estimate (however, this time scale clearly depends on system size, since for a large system different subsystems would be undergoing MB-MB transition events at different times). Thus, MB-MB transitions (the crossings from one island to another and which last 10-20\% of $t^{*}$ ) are fast events compared to the times for the exploration of the MBs. The study of MB-MB transition events has been done previously [18], revealing the decisive role of large compact clusters of medium-range-mobility particles (which we call $d$ clusters). These clusters are responsible for the $\alpha$ relaxation (completed after a few of such events) and represent potential candidates for the cooperatively relaxing regions of Adam and Gibbs [18]. The compact nature of these events relevant to the $\alpha$ relaxation would be compatible with the geometrical structure of the dynamics correlations at large time scales on the order of $\tau_{\alpha}$, as indicated in an inhomogeneous mode-coupling theory (at variance from the less dense structures, compatible with stringlike motions, expected at the shorter time scales of $\beta$-relaxation time) [22]. We also show in Fig. 1 (middle) the distance matrix calculated for the same trajectory but with the inherent structures instead of the instantaneous or real configurations in order to filter out the randomizing effect introduced by the thermal vibrations [3] (this subtracting of the thermal motions makes the IS approach useful to better determine the relative contribution of the different events to the structural relaxation and thus we shall employ such method in most of our following work). The ISs represent the closest local minimum of each instantaneous configuration within the potential-energy surface of the system. Thus, each instantaneous structure represents a thermal induced deformation of its corresponding IS. To locate the ISs we minimized the instantaneous configurations by means of the conjugate gradient method (we had not applied this method for the MD simulations of this system in our previous works within the NVE ensemble). Here we can note that the results are analogous at both levels of description. However, since the (computationally more expensive) IS method is more efficient for identifying dynamical events (given the fact that the structural changes are free form the masking effect of the thermal fluctuations) we shall employ it for certain parts of the following analysis instead of simply using the real dynamics.

The democratic particles that comprise the $d$ clusters that trigger MB-MB transitions were defined as that whose mobility was greater than $r_{t h}=0.3$ within the time interval $\theta$ $=40$ at this temperature for the real dynamics (the chosen cutoff represents roughly the value of $r$ when the distribution of particle displacements within a time interval characterized by a MB transition event exceeds the van Hove function, that is, the time-averaged behavior), and its fraction was represented by the function $m(t, \theta)[18]$. Thus, for the system size under study we found that on the order of 40-60 particles were involved in a $d$ cluster [18].

We also display in Fig. 1 (bottom) the function $\delta^{2}(t, \theta)$, the system-averaged-squared displacement of the particles within a time interval $\theta=40$ (gray line). This function is defined as

$$
\begin{gathered}
\delta^{2}(t, \theta)=R^{2}(t-\theta / 2, t+\theta / 2) \\
\delta^{2}(t, \theta)=\frac{1}{N} \sum_{i=1}^{N}\left|\mathbf{r}_{i}(t-\theta / 2)-\mathbf{r}_{i}(t+\theta / 2)\right|^{2} .
\end{gathered}
$$

Thus, $\delta^{2}(t, \theta)$ is $R^{2}\left(t^{\prime}, t^{\prime \prime}\right)$ measured along the diagonal $t^{\prime \prime}$ $=t^{\prime}+\theta$ and hence the average of this quantity over different start times $t$ gives the usual mean-squared displacement for time lag $\theta$. We again note that the time scale $\theta=40$ is significantly smaller than the $\alpha$-relaxation time $\left(\tau_{\alpha} \approx 700\right)$ but still sufficiently larger than the time of the microscopic vibrations $[=O(1)]$. For some trajectories it is enough to calculate this function with the instantaneous (real) configurations, as can be seen in Ref. [18]. In some cases, however, the masking effect of the thermal vibrations obscures a bit the results and it is better to calculate the function at the inherent dynamics (over the minimized configurations), as we have done here. A comparison of this $\delta^{2}(t, \theta)$ with Fig. 1 (top and middle) shows that $\delta^{2}(t, \theta)$ is showing pronounced peaks exactly when the system leaves an MB. Thus, we see that changing the $\mathrm{MB}$ is indeed associated with a rapid motion as measured in $\delta^{2}(t, \theta)$. We define democratic particles as those which in the time interval $\theta=40$ have moved more than $r_{t h}=0.25$ for the inherent dynamics (we use a lowest threshold than in the real dynamics since in the inherent dynamics the contribution of the thermal fluctuations to the particles displacements has been removed; we also note that while this threshold value is arbitrary, the results are robust with respect to changes of such value from around 0.1 to values much larger than 0.25 ) and denoted the fraction of such particles by $m(t, \theta)$. In Fig. 1 (bottom), we have also included such fraction of democratic particles $m(t, \theta)$ at the inherent dynamics as a function of time (black bars). The comparison with $\delta^{2}(t, \theta)$ shows that the fraction of these particles is indeed large whenever the $\delta^{2}(t, \theta)$ increases rapidly. This fraction is on the order of $30-50 \%$ of all the particles and, thus, significantly larger than one would expect from the integration of (the self part of) the van Hove function $4 \pi r^{2} G_{\mathrm{s}}(r, \theta)$ (where $G_{\mathrm{s}}(r, \theta)=N^{-1} \sum_{i=1}^{N}\left\langle\delta\left(r-\left|\mathbf{r}_{i}(\theta)-\mathbf{r}_{i}(0)\right|\right)\right\rangle$ is the averaged distribution of particle displacements for time lag $\theta$ ) from $r_{t h}$ to infinity in the inherent dynamics that gives $\int_{0.25}^{\infty} 4 \pi r^{2} G_{s}(r, \theta) \sim 0.09$. Finally, we mention that the temperature we use, $T=0.5$, is one at which the MB structure of the potential-energy landscape is valid (which coincides with the prevalence of the dynamical heterogeneities, roughly between around $T=0.6$ to $\left.T_{C}=0.435\right)$. If we change temperature within such time interval the results are similar, improving a bit as $T$ diminishes.

\section{RESULTS}

\section{A. In-time heterogeneity of the correlation functions}

This section is devoted to study in detail the time evolution of the transport properties in the above mentioned archetypical model glass former (the binary Lennard-Jones mixture) in order to determine to which extent the dynamics for 


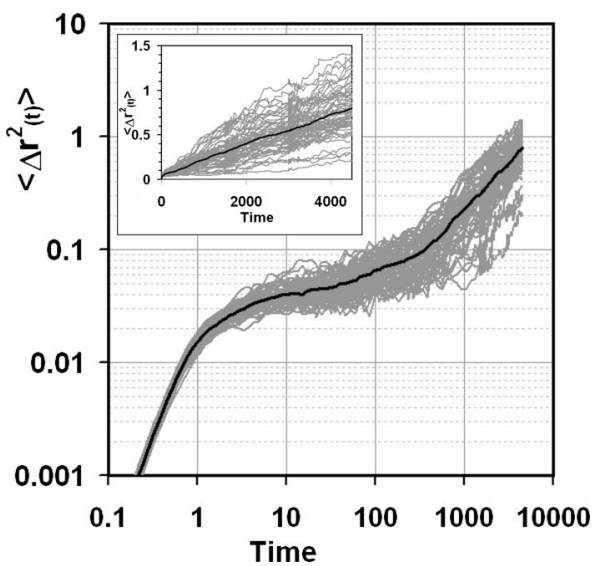

FIG. 2. The squared displacement function for a single trajectory, $R^{2}(0, t)$. The black solid line is this function calculated for a system of $N=8000$ and $T=0.5$. The gray solid lines correspond to the calculation of such function for each of the 64 boxes in which the system of $N=8000$ was divided. The inset shows a linear plot of the same data. The average behavior of the gray curves coincides with that of the large system.

small systems or within regions of a large system instead of being gradual is heterogeneous in time. To that end, one of the simplest time-dependent correlation functions to focus on is the mean-squared displacement, MSD, function

$$
\left\langle r^{2}(t)\right\rangle=\frac{1}{N} \sum_{i=1}^{N}\left|\mathbf{r}_{i}(t)-\mathbf{r}_{i}(0)\right|^{2},
$$

where $\mathbf{r}_{i}(t)$ is the position of particle $i$ at time $t$. To gather enough statistics to yield a smooth curve, it is necessary to calculate this function (usually for a large system size) averaging over large times (much larger than the $\alpha$-relaxation time) or over many independent initial configurations. This requirement is more stringent when one calculates the function for a small system size. However, this averaging washes out the fingerprints of the time heterogeneity, as we shall see later on. Thus, here we take a different approach and calculate the mean-squared displacement for a single run (a single trajectory for a given single initial configuration) of length larger than the $\alpha$-relaxation time (many of the plots we shall show will thus be run dependent, but will illustrate typical behaviors verified in many single runs). In other words, we are going to plot the first row (or column) of the distance matrix $R^{2}\left(t^{\prime}, t^{\prime \prime}\right)$, that is the function $R^{2}(0, t)$ (here, however, we shall take configurations more closely spaced than that used before for the distance matrix). This function thus measures the extent to which the given trajectory of the system has moved away at time $t$ from its initial position at time $t$ $=0$ (as measured by the squared displacement averaged over all the particles). When we plot such function for a large system, the behavior is not very different from that of the usual MSD plots for such system size, albeit with a bit poorer statistics (see Fig. 2, which is very similar to the MSD curve reported in the literature for this temperature by Kob and Andersen [33]). Here in Fig. 2 we show such results for $N=8000$ and a low temperature close to $T_{C}, T=0.5$. The
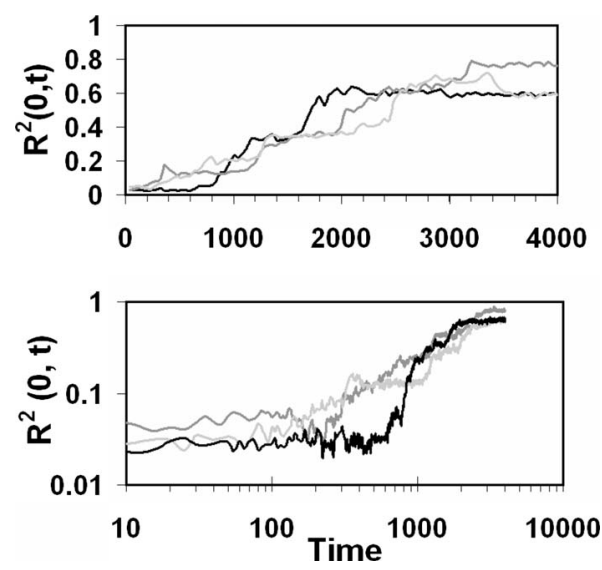

FIG. 3. Top: the solid black line depicts the function $R^{2}(0, t)$ for a system of $N=150$, corresponding to the same trajectory as that of Fig. 1 displayed on a linear scale to better evidence the jumps presented by the curve. The times when the curve exhibits important jumps coincide with that of the islands transitions of Fig. 1. The other two curves display the same function for single trajectories in other two independent $N=150$ systems. Bottom: the same curves but now displayed in a log-log fashion.

(logarithmic in time) function $R^{2}(0, t)$ shows at first a ballistic regime (slope 2 ) and then a plateau (corresponding to the caging of the particles by their first neighbors), followed by the typical diffusive regime (slope 1). The time scale of maximum inhomogeneous behavior, $t^{*}$ is located at end of the plateau beginning of the diffusive regime, around the onset of the $\alpha$ relaxation [9]. However, if the system presents dynamical heterogeneities and $d$-cluster events, we expect that the evaluation of $R^{2}(0, t)$ for a small system or for a small subsystem (a small region within a large system) shall present clearly different features. This is precisely the case, as can also be seen from Fig. 2 gray lines and in Fig. 3. In Fig. 2 (gray curves) we show the same function $R^{2}(0, t)$ (calculated at the same times) but for 64 equal-size sub-boxes (of around 125 particles each) in which we divided the same large $N=8000$ system. We can clearly note that the dynamics at distinct portions of the large system (within the different subsystems) are quite different, with regions that are much faster or slower than the average behavior of the whole system (we note that if we average the curves of the different subsystems we produce a curve that resembles very well that for the large system).

A similar situation would also arise for a small isolated system. We show in Fig. 3 (top), black line, the function $R^{2}(0, t)$ calculated for an isolated small-size system $(N$ $=150)$. This curve corresponds to the same system of Fig. 1 and to the same trajectory, plotted in a linear time scale. We also show in such graph the same function calculated for other single trajectories in systems of $N=150$. From this plot, and also from the ones that correspond to the subsystems already analyzed (Fig. 2, gray curves), we can learn an instructive detail: the $R^{2}(0, t)$ function is not a smooth curve like the one for the large system of Fig. 2 (black line) or like the typical MSD plots, but shows instants when it increases markedly. In Fig. 3 (top) we have also included other two $R^{2}(0, t)$ functions calculated for other two single runs in a 
system of $N=150$. All these curves illustrate the typical behavior: the function shows large plateaus and relatively fast increases (steps). In Fig. 3 (bottom), in turn, we have plotted the same functions as that of Fig. 3 (top) but in a log-log plot. Even with the poor statistics of single runs we can appreciate the ballistic regime, the plateau and the diffusive regime. However we can note that the diffusive slope 1 regime is followed in zigzag given the plateau-jump or stepwise nature of the curves (we also note that if we average many curves like the ones shown, we shall recover a smooth slope 1 linear regime). This behavior of the squared displacement function constitutes a clear indication that the dynamics is heterogeneous in time, a fact that has not been recognized previously in former studies of large systems. This heterogeneous nature of the local dynamics (at the subsystem level) reflects itself in a metabasin structure of the potential-energy landscape such as that of Fig. 1, while the island structure would disappear if the distance matrix were calculated for a large $(N=8000)$ system. The jumps in $R^{2}(0, t)$ in Fig. 3 signaling the metabasin transitions (please compare the jumps in the black line of Fig. 3 with the island transitions of Fig. 1 ) indicate the sparse and rapid nature of the $d$-cluster events which are responsible for the advancement of the relaxation (while the rest of the time the trajectory does not progress significantly). In turn, the fact that the function $R^{2}(0, t)$ calculated for a large system lacks any step-wise nature, as previously seen, is due to the fact that in the different subsystems of the large system the times of the occurrence of the $d$ clusters are different. Thus, while the $R^{2}(0, t)$ curves for each of the subsystems evolve by means of jumps, the averaging over all the subsystems produces a curve with a nice plateau and diffusive regime more typical of the MSD one is used to see in the literature (the smoothness of the curve increasing as $N$ grows toward the thermodynamic limit). The same averaging out of the heterogeneous behavior occurs when we average the $R^{2}(0, t)$ functions of many different independent small isolated systems or when one calculates it for a very long run (by averaging the behaviors over many different time intervals within such trajectory). A note of caution must be placed, however, regarding the comparison of the behavior of small isolated systems and small subsystems within a large one which we studied above. Given the fact that the sub-boxes in which we divided the large system have been arbitrarily placed, they might cut the different $d$ clusters that would occur within the large system which might span through more than one single box. Thus, the distance matrix for the sub-boxes, while still heterogeneous and presenting an island structure, would be less neat than the ones for a small isolated system [a loss of quality that also would affect the magnitude of the jumps in the function $R^{2}(0, t)$ ]. Thus, while both kinds of small systems produce similar (time) heterogeneity, in most of the following we shall employ small isolated systems to better single out such behavior.

The other typical correlation function in this context is the incoherent intermediate scattering function $F_{s}(q, t)$, which is usually calculated at the wave-vector $q$ corresponding to the maximum in the structure factor

$$
F_{s}(q, t)=N^{-1} \sum_{j=1}^{N}\left\langle\exp \left\{i \mathbf{q} \cdot\left[\mathbf{r}_{j}(t)-\mathbf{r}_{j}(0)\right]\right\}\right\rangle .
$$
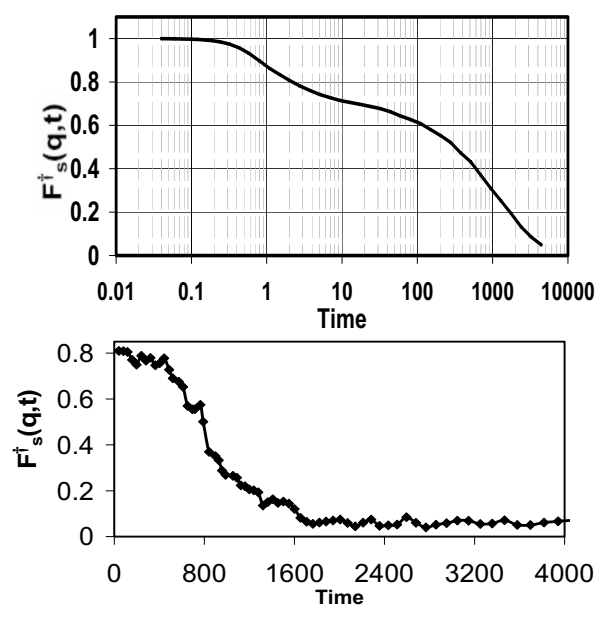

FIG. 4. Top: self-intermediate scattering function $F_{s}^{\dagger}(q, t)$ for a system of $N=8000$ at $T=0.5$ (the same trajectory as that of Fig. 2 . Bottom: $F_{s}^{\dagger}(q, t)$ for a system of $N=150$ and the same temperature (corresponding to the trajectory of Fig. 1 and the black lines of Fig. 3).

Such correlation function accounts for the loss in time of the memory of the initial structural configuration and exhibits a first decay, corresponding to the caging regime, and a second decay characteristic of the $\alpha$ relaxation. Here, we again shall not calculate the typical $F_{s}(q, t)$ function, but we shall compute the function $F_{s}^{\dagger}(q, t)$, which is the same mathematical formulation but calculated for a single trajectory of length on the order of the $\alpha$-relaxation time (from a single initial structure). This is depicted in Fig. 4 (top) for a large system size $(N=8000)$. However, if the system presents dynamical heterogeneities and $d$-cluster events we expect, as before, that when we evaluate such function for a small system or for a small subsystem (a small region within a large system) it shall present a roughly step-wise behavior. This is precisely the case, as we can also learn from Fig. 4 (bottom) where we show the results for the same $N=150$ system and same trajectory as that of Fig. 1. From this figure (shown with a linear time axis instead of the usual logarithmic one to stress this point), we can also see that the curve is not smooth (like the one for the large $N=8000$ system), but shows instants when it decays markedly, a behavior consistent with the stepwise nature of the $R^{2}(0, t)$ function of Fig. 3 , and a clear indication that the dynamics is quite heterogeneous in time.

To emphasize this point, we introduce now the function $\Delta F_{s}^{\dagger}(q, t, \theta)$ as $F_{s}^{\dagger}(q, t+\theta / 2)-F_{s}^{\dagger}(q, t-\theta / 2)$, which gives the change in the scattering function $F_{s}^{\dagger}(q, t)$ at time $t$ and for time interval $\theta=40$. The behavior of this function is displayed in Fig. 5 (top) for a typical trajectory for a system of $N=150$ particles [the same as that of Fig. 1 and the black line of Fig. 3 and Fig. 4 (bottom)]. We can note that $\Delta F_{s}^{\dagger}(q, t, \theta)$ shows the presence of peaks which signal the abrupt decays in $F_{s}^{\dagger}(q, t)$ and which coincide with that of the $R^{2}(0, t)$ function and the metabasin transitions ( $d$ clusters). This result makes evident the fact that the dynamics is very heterogeneous in time since most of the decay of the correlation function is performed at such events, the rest of times exhibiting poor contributions. 

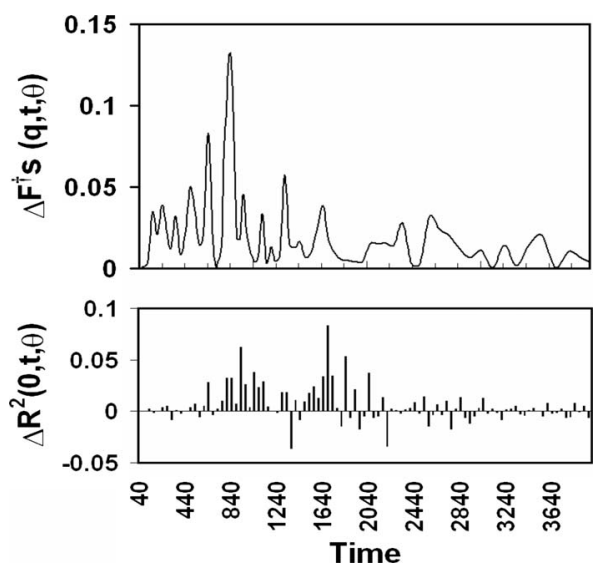

FIG. 5. Top: the function $\Delta F_{s}^{\dagger}(q, t, \theta)$; Bottom: plot of $\Delta R^{2}(0, t, \theta)$, in this case, evaluated at the inherent dynamics.

We also return now to real space to define the function $\Delta R^{2}(0, t, \theta)=R^{2}(0, t+\theta / 2)-R^{2}(0, t-\theta / 2)$, that is, the change in the squared displacement function $R^{2}(0, t)$ at time $t$ and evaluated for time interval $\theta=40$ (here we have used the inherent dynamics to filter out the randomizing effect of the thermal energy). In other words, we wish to investigate to which extent the squared displacement of a single trajectory is advanced at each time of the trajectory. Direct inspection of Fig. 5 (bottom) confirms our conclusion that the MB transitions ( $d$ clusters) perform great contributions while the rest of the time the MSD is not significantly advanced.

\section{B. Assessing the relevance of the different kinds of dynamical events}

This section is devoted to determining the role of the $d$ clusters or MB transitions events and of the MB exploration events to the structural relaxation of the system. We shall show that the former are almost exclusively responsible for the structural relaxation, while the latter do not contribute appreciably. To better quantify this point we analyzed $60 \mathrm{MD}$ trajectories of length $t=4000$ at $T=0.5$ for an isolated system of $N=150$ and recorded 100 equally spaced configurations for each trajectory. To locate MB transitions and the corresponding $d$ clusters we employed an automatic simplified method: for each trajectory in the real dynamics we calculated the function $m(t, \theta)$, that is, the fraction of particles that moved more than $r_{t h}=0.3$ within each of the $\theta=40$ time intervals (between contiguously recorded configurations). Whenever this value is larger than 0.3 we say that the trajectory has undergone a $d$-cluster event. This approximate method is based on our observation from many different trajectories of the minimum number of particles that typically move in such an event at this temperature [18] (we recall that the integration of the van Hove function from $r_{\text {th }}$ to infinity for the real dynamics gives only $\sim 0.16[18]$ ). With this method we find a total number of $168 d$ clusters, roughly an average of three events per trajectory. We now calculate the average change in the squared displacement during a $d$ cluster. That is, we calculate the value of $\left\langle\Delta R^{2}(0, t, \theta)\right\rangle$ $\left.=\left\langle R^{2}(0, t+\theta / 2)\right)-R^{2}(0, t-\theta / 2)\right\rangle$, where the mean-squared

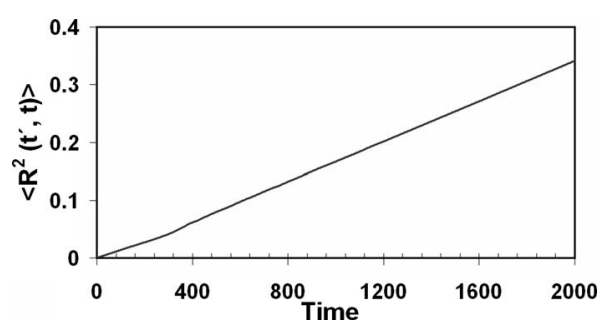

FIG. 6. The function $\left\langle R^{2}\left(t^{\prime}, t\right)\right\rangle$ as calculated by averaging over 60 different trajectories (in a linear plot). $T=0.5$ and we have made use of the inherent structure dynamics. The lack of an inertial (ballistic) and a caging regime such as that usually seen in a typical MSD curve is due to the fact that the inherent formalism is being used [34] (these regimes do in fact emerge at the real dynamics).

displacement is evaluated for time intervals $[t-\theta / 2, t$ $+\theta / 2]$, with $\theta=40$, only when a $d$ cluster occurs and the average is performed over time and over all the 60 trajectories. That is, we average the advancement of the squared displacement function over the $168 d$ clusters found. We employ now the IS formalism in order to be free of the masking effect of the thermal vibrations, thus being able to better discriminate the average contribution of these kind of events. We do so since, as shall be made clearer later on in Fig. 6 and Fig. 7, the motion of the particles comprising the $d$ clusters does not depend too much on the kind of approach used, real or inherent dynamics, while the (averaged over all time intervals of length $\theta$ ) advancement in the squared displacement of the trajectory is notably much larger for the real dynamics (due mainly to the thermal motions). This last value constitutes basically the contribution to the squared displacement of the time intervals when no $d$ cluster occurs, given the sparse location of $d$ clusters. Thus, the thermal fluctuations would make the relative contribution of the $d$ clusters look a bit less relevant. With the above described method, we find that an average increment in the squared displacement of the trajectory of 0.09 occurs for a $d$ cluster during a $t=40$ time interval in the inherent dynamics. For comparison, we plot in Fig. 6 the function $\left\langle R^{2}\left(t^{\prime}, t\right)\right\rangle$ averaged over the 60 different trajectories (in a linear plot). This function calculates the squared displacement for a time interval of length $t$ as calculated by moving the beginning of the

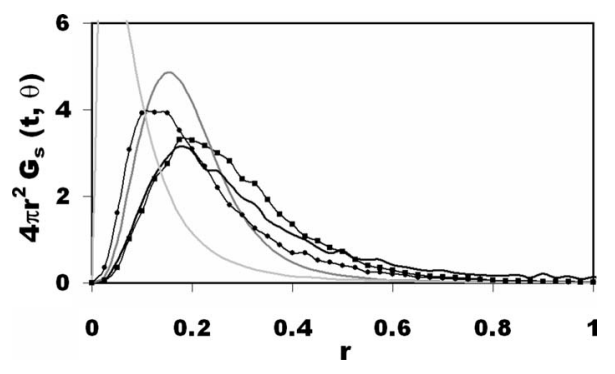

FIG. 7. Van Hove function for time $t=40$ for the instantaneous configurations or real dynamics (solid dark gray line) and for the inherent dynamics (solid light gray line). Particle displacements distribution for time intervals wherein a $d$ cluster occurs (of length $t$ =40) for the real dynamics (line with filled squares) and for the inherent dynamics (line with filled circles). Van Hove function for $t=\tau_{\alpha} \approx 700$ (solid black line). 
time interval $t^{\prime}$ over all times for each of the 60 trajectories, evaluated at the inherent dynamics. The outcome is clearly very similar to the usual MSD plot and the behavior at large times is linear, as demanded by a diffusive regime. We find that the value of this function at time $t=40$ is only 0.007 , an order of magnitude less than for a $d$ cluster. We can also learn that the average advancement of the trajectory during a $d$ cluster (within a time interval of $t=40$ ) is close to that performed during the whole time scale of the $\alpha$ relaxation, $\tau_{\alpha} \approx 700$. We note that in the calculation of the curve of Fig. 6 , the contribution of the different $d$ clusters that occur for the different trajectories (many of them presenting a $d$ cluster before $\tau_{\alpha}$ ) is not subtracted. Thus, this fact indicates that the squared displacement is mostly advanced by the $d$ clusters, while the rest of the time, the trajectory contributes quite modestly.

Similar information can be obtained from the study of the van Hove function and the displacement distributions. The van Hove function $4 \pi r^{2} G_{s}(r, t)$ gives the probability for a particle to be located at a distance $r$ from the origin after time $t$. In Fig. 7 we display this function evaluated at time $t=40$ and $t=\tau_{\alpha} \approx 700$ for the real dynamics (this function gives the averaged displacement of the particles for a time interval of length $t$, as averaged over all the 100 time intervals of all 60 trajectories). We also show this function but evaluated only at the $168 d$ clusters found (for time intervals of length $t=40$ containing a $d$ cluster event). Again we can see that the displacement distribution for the $d$ clusters is very different from the behavior of the van Hove function for $t=40$ (clearly displaced to the right and thus indicating a great enhancement in particle motion) and similar to that function evaluated at $\tau_{\alpha}$ (we again remind that in this last case the contribution of the $d$ clusters is also included since it is averaged over all times). Thus, this reinforces the notion that the MB exploration events do not contribute significantly to the long-time structural relaxation, which is almost exclusively governed by the $d$ clusters. We have also included the van Hove function for $t=40$ evaluated at the inherent dynamics and the particle displacements distribution for time intervals of length $t=40$ wherein a $d$ cluster occurs also for the inherent dynamics. We can thus see that the functions at the real and inherent dynamics give similar information but that the removal of the thermal fluctuations at the IS level provides neater results. The curves of the van Hove function and the displacement distributions at the $d$-cluster events intersect at around $r=0.25$ and $r=0.10$ for the real and inherent dynamics, respectively. Thus, the $d$ clusters at the IS level are more evident than at the real dynamics. If we calculate the excess of the distribution curve for the particle displacements at the $d$ clusters with respect to the van Hove function (integrating both curves from the intersection point to infinity and subtracting them) we find a value of $\approx 0.45$ at the IS level while it drops to roughly 0.3 in the real dynamics. This gives further support to our former choice of the inherent formalism (which subtracts the thermal motions) to calculate the relative contribution of the $d$-cluster events to the advancement of the correlation functions, namely, $R^{2}(0, t)$ and $F_{s}^{\dagger}(q, t)$.

\section{CONCLUSIONS}

Different studies have suggested that the dynamics of glass-forming systems is heterogeneous both in space and in time, since for the different regions of the system the trajectory is locally confined for long times within metabasins of the potential-energy surface to experience abrupt metabasin transitions characterized by significant molecular reorganizations or $d$ clusters. In the present work we confirm this picture wherein the last mentioned relaxation events play a major role in the long-time dynamics. We show that the different correlation functions (the intermediate scattering function and the particles squared displacements for a single trajectory) for small systems or for small regions of a large system present quite a different behavior than that typically obtained when evaluated over a whole large system, displaying abrupt changes that reveal the presence of major relaxation events. We also show that relevant changes in such functions are only produced whenever the system suffers a $d$ cluster, while the rest of the dynamics does not contribute significantly. Thus, such functions are mainly advanced by the indicated events and the average contribution of a $d$ cluster is shown to be close to that of the whole $\alpha$-relaxation time scale. In this sense, these findings reveal the metabasin transition event ( $d$ cluster) as the basic mechanism responsible for the long-time diffusion in the supercooled systems under study.

\section{ACKNOWLEDGMENTS}

Financial support from ANPCyT, SeCyT, and CONICET is gratefully acknowledged. G.A.A., M.A.F., and L.M.A. received additional support from CONICET. R.A.M. received additional support from CIC.
[1] C. A. Angell, J. Non-Cryst. Solids 131-133, 13 (1991).

[2] M. D. Ediger, Annu. Rev. Phys. Chem. 51, 99 (2000).

[3] P. G. Debenedetti and F. H. Stillinger, Nature (London) 410, 259 (2001).

[4] M. D. Ediger, C. A. Angell, and S. R. Nagel, J. Phys. Chem. 100, 13200 (1996).

[5] W. Götze, J. Phys.: Condens. Matter 11, A1 (1999).

[6] Proceedings of the Fourth International Discussion Meeting on Relaxations in Complex Systems, 2002, edited by K. L.
Ngai [J. Non-Cryst. Solids 307-310, 1 (2002)].

[7] G. Adam and J. H. Gibbs, J. Chem. Phys. 43, 139 (1965).

[8] K. Schmidt-Rohr and H. W. Spiess, Phys. Rev. Lett. 66, 3020 (1991).

[9] C. Donati, J. F. Douglas, W. Kob, S. J. Plimpton, P. H. Poole, and S. C. Glotzer, Phys. Rev. Lett. 80, 2338 (1998).

[10] R. Richert, J. Phys.: Condens. Matter 14, R703 (2002).

[11] E. R. Weeks, J. C. Crocker, A. C. Levitt, A. Schofield, and D. A. Weitz, Science 287, 627 (2000). 
[12] W. K. Kegel and A. van Blaaderen, Science 287, 290 (2000).

[13] W. Kob, C. Donati, S. J. Plimpton, P. H. Poole, and S. C. Glotzer, Phys. Rev. Lett. 79, 2827 (1997).

[14] S. Butler and P. Harrowell, J. Chem. Phys. 95, 4454 (1991).

[15] M. T. Cicerone, F. R. Blackburn, and M. D. Ediger, J. Chem. Phys. 102, 471 (1995).

[16] G. A. Appignanesi, M. A. Frechero, and R. A. Montani, Physica A 329, 41 (2003).

[17] G. A. Appignanesi, M. A. Frechero, L. M. Alarcón, J. A. Rodriguez Fris, and R. A. Montani, Physica A 339, 469 (2004).

[18] G. A. Appignanesi, J. A. Rodriguez Fris, R. A. Montani, and W. Kob, Phys. Rev. Lett. 96, 057801 (2006).

[19] B. Doliwa and A. Heuer, Phys. Rev. E 67, 030501(R) (2003).

[20] M. Vogel, B. Doliwa, A. Heuer, and S. C. Glotzer, J. Chem. Phys. 120, 4404 (2004).

[21] J. A. Rodriguez Fris, G. A. Appignanesi, E. La Nave, and F. Sciortino, Phys. Rev. E 75, 041501 (2007).

[22] G. Biroli, J.-P. Bouchaud, K. Miyazaki, and D. R. Reichman, Phys. Rev. Lett. 97, 195701 (2006).

[23] R. A. L. Vallée, W. Paul, and K. Binder, J. Chem. Phys. 127, 154903 (2007).

[24] This seems reasonable from the results of the work of Ref. [18]. However, a more careful study of the mean $d$-cluster size as a function of temperature within the supercooled regime for the binary Lennard-Jones system (by studying statistically the $d$ clusters present within large systems) is demanded and is presently being carried out. Our preliminary results indicate that, for instance, for $T=0.5$ the mean $d$-cluster size is not far from $N=150$.

[25] G. A. Appignanesi, J. A. Rodriguez Fris, and M. A. Frechero, Phys. Rev. Lett. 96, 237803 (2006).

[26] A. Widmer-Cooper and P. Harrowell, J. Chem. Phys. 126, 154503 (2007)

[27] A. Widmer-Cooper, P. Harrowell, and H. Fynewever, Phys. Rev. Lett. 93, 135701 (2004).

[28] A. Widmer-Cooper, H. Perry, P. Harrowell, and D. Reichman, Nat. Phys. 4, 711 (2008).

[29] A. Heuer, B. Doliwa, and A. Saksaengwijit, Phys. Rev. E 72, 021503 (2005).

[30] We use reduced units as in Ref. [18] $\left(\sigma_{A A}\right.$ and $\epsilon_{A A}$ as units of length and energy, respectively), and we measure time in units of $\left(m \sigma_{A A}^{2} / 48 \epsilon_{A A}\right)^{1 / 2}$.

[31] W. Kob and H. C. Andersen, Phys. Rev. E 51, 4626 (1995).

[32] I. Ohmine, J. Phys. Chem. 99, 6767 (1995).

[33] W. Kob and H. C. Andersen, Phys. Rev. Lett. 73, 1376 (1994).

[34] T. B. Schrøeder and J. C. Dyre, J. Non-Cryst. Solids 235-237, 331 (1998) 